\title{
Efecto del uso de la herramienta "realidad aumentada" en el rendimiento académico de estudiantes de Educación Básica
}

\author{
Effect of the use of the tool "augmented reality" \\ in the academic performance of students of Basic Education \\ Efeito do uso da ferramenta "realidade aumentada" \\ no desempenho acadêmico de alunos do Ensino Fundamental \\ José David López-García ${ }^{a}$, Deidy Gutiérrez-Niño*b
}

aMaestro en Informática educativa; Corporación Universitaria Minuto de Dios - Bogotá,

bMaestra en informática educativa, Corporación Universitaria Minuto de Dios - Bogotá.

Forma de citar: López, J. \& Gutiérrez, D. (2018). Efecto del uso de la herramienta "Realidad aumenta" en el rendimiento académico de estudiantes de Educación Básica. Perspectivas, 3(1). 6-12

Recibido: julio 22 de 2017

Aceptado: octubre 10 de 2017

\section{Palabras clave}

Realidad aumentada, estrategias didácticas, regiones naturales, enseñanza apoyada por Tic.

* Autor para correspondencia deidygutnin@gmail.com
Resumen: La comprensión de los fenómenos naturales, las características de los contextos y de las relaciones espacio-temporales, puede verse afectada cuando no se emplean los recursos y materiales y se aprovechan espacios innovadores que se adecuen a las demandas de los estudiantes durante el desarrollo didáctico de los programas del currículo. Debido a su gran riqueza y complejidad, el tema de las regiones naturales de Colombia es uno de los contenidos para cuya comprensión los estudiantes presentan recurrentemente serias dificultades. Motivados por esta situación, el propósito de este estudio ha consistido en evaluar el efecto de la implementación de la herramienta de Realidad Aumentada (RA) en el rendimiento académico de los estudiantes de educación básica. En cuanto a la metodología, se enmarca en un estudio de tipo cuasi experimental, que partió de una búsqueda de información, observación directa y aplicación de pruebas de entrada y salida a estudiantes organizados en dos grupos de trabajo (experimental y control). El contexto del estudio fue el Colegio Virginia Gutiérrez de Pineda, ubicado en la ciudad de Bogotá. Se encontró que los estudiantes del grupo experimental evidenciaron un

https://doi.org/10.22463/25909215.1464 
aprendizaje significativo, asimilaron la información teórica con mayor facilidad y mejoraron los resultados en la prueba de salida con respecto a la prueba de entrada. En función de estos resultados, se recomienda invertir recursos económicos, tiempo y personal para capacitación en tecnologías de realidad aumentada y diseño de material didáctico que permita optimizar los procesos de aprendizaje en los estudiantes.

\section{Keywords}

Augmented reality, teaching strategies, natural regions, teaching supported by Tic.

\section{Palavras chave}

Realidade aumentada, estratégias de ensino, regiões naturais, ensino apoiado por Tic.

\begin{abstract}
The understanding of natural phenomena, the characteristics of contexts and spatio-temporal relationships, can be affected when resources and materials are not used and innovative spaces are used that adapt to the demands of students during the didactic development of curriculum programs. Due to its great richness and complexity, the subject of the natural regions of Colombia is one of the contents for whose understanding the students present recurrently serious difficulties. Motivated by this situation, the purpose of this study was to evaluate the effect of the implementation of the Augmented Reality (AR) tool on the academic performance of basic education students. Regarding the methodology, it is part of a quasi-experimental study, which started with a search for information, direct observation and application of entrance and exit tests to students organized in two work groups (experimental and control). The context of the study was the Virginia Gutierrez de Pineda School, located in the city of Bogotá - Colombia. It was found that the students of the experimental group showed significant learning, assimilated the theoretical information more easily and improved the results in the exit test with respect to the entrance test. Based on these results, it is recommended to invest economic resources, time and personnel for training in augmented reality technologies and design of teaching materials that optimize the learning processes in students.
\end{abstract}

Resumo: A compreensão dos fenômenos naturais, das características dos contextos e das relações espaço-temporais, pode ser afetada quando recursos e materiais não são utilizados e são utilizados espaços inovadores que se adaptam às demandas dos alunos durante o desenvolvimento didático de programas curriculares. Devido à sua grande riqueza e complexidade, o tema das regiões naturais da Colômbia é um dos conteúdos para cuja compreensão os alunos apresentam dificuldades sérias recorrentes. Motivado por esta situação, o objetivo deste estudo foi avaliar o efeito da implementação da ferramenta Realidade Aumentada (RA) no desempenho acadêmico de estudantes do ensino fundamental. Quanto à metodologia, faz parte de um estudo quaseexperimental, que iniciou com a busca de informações, observação 
direta e aplicação de testes de entrada e saída aos alunos organizados em dois grupos de trabalho (experimental e controle). O contexto do estudo foi a Escola Virginia Gutiérrez de Pineda, localizada na cidade de Bogotá - Colômbia. Verificou-se que os alunos do grupo experimental apresentaram aprendizado significativo, assimilaram mais facilmente as informações teóricas e melhoraram os resultados no teste de saída em relação ao teste de entrada. Com base nesses resultados, recomendase investir recursos econômicos, tempo e pessoal para treinamento em tecnologias de realidade aumentada e design de materiais didáticos que otimizem os processos de aprendizagem dos alunos.

\section{Introducción}

La Realidad Aumentada (RA) es una tecnología emergente, la cual está aplicando en las áreas de la publicidad, el comercio y especialmente en la educación donde tiene un alto potencial de desarrollo y ofrece un gran valor agregado en los procesos de enseñanza y aprendizaje.

La RA consiste en "añadir gráficos virtuales, en tiempo real, al campo de visión de una persona. $\mathrm{Su}$ finalidad es superponer al entorno real información que permita enriquecerla. Se diferencia de la realidad virtual en que mientras ésta pretende reemplazar el mundo real, la realidad aumentada lo que hace es complementarla". (Catalá, Jaén y Mocholí,2008: 143).

A partir del documento de la Fundación Telefónica se define la RA como una nueva forma de ver los objetos del mundo real que se ven enriquecidos con información digital. Es un proceso en el que se mejoran los datos del mundo físico con información digital mediante la superposición de imagen, audio, y modelos 3D, brindando"una experiencia de usuario en el que se diluyan las barreras entre lo real y lo virtual" (Fundación Telefónica, 2012).

Fombona, Pascual y Madeira (2012) basan su definición sobre todo en las herramientas técnicas (hardware o dispositivos) que permiten registrar elementos de la realidad e integrar sobre ello sistemas de RA. De este modo, afirman que «la realidad aumentada amplía las imágenes de la realidad, a partir de su captura por la cámara de un equipo informático o dispositivo móvil avanzado que añade elementos virtuales para la creación de una realidad mixta a la que se le han sumado datos informáticos» (Fombona, Pascual y Madeira, 2012:203).

En el campo de la educación se han desarrollado diferentes trabajos de implementación de la RA, como por ejemplo el llevado a término por Abdud (2012) en México, que propone la creación de objetos de aprendizaje basados en esta tecnología para un curso de medicina y que como conclusión propone un modelo para el manejo de la interacción entre el ser humano y la computadora.

La realidad aumentada está relacionada con la tecnología realidad virtual, más extendida y conocida en las sociedades actuales. Ambas presentan características comunes, como por ejemplo la inclusión de modelos virtuales gráficos 2D y 3D en el campo de visión del usuario. La principal diferencia se halla en que la realidad aumentada no reemplaza el mundo real por uno virtual, sino al contrario, mantiene el mundo real que ve el usuario complementándolo con información virtual superpuesta ala real. El usuario nunca pierde el contacto con el mundo real que tiene al alcance de su vista, pero al mismo tiempo puede interactuar con la información virtual superpuesta (Basogain, Olabe, Espinosa, Rouèche y Olabe:2007).

La realidad aumentada en contextos educativos ha sido implementada con éxito en varias experiencias. Desde la realización de videos (Rockwell y Singleton, 2007), el uso de videoconferencias (Suduc, Bizoi y Gorghiu, 
2010) han surgido ideas novedosas e interesantes sobre cómo realizar videos atractivos y orientados alaprendizaje. Cherrett, Wills, Price, Maynard y Dror (2009) describen por su parte la eficacia de las presentaciones y ofrecen un análisis acerca de la motivación del uso de videos como elemento motivador en las clases. En contextos latinos se encuentran experiencias innovadores a nivel de educación primaria en trabajos como el de De Pedro y Martínez (2012), cuyos aportes vislumbran a corto plazo un amplio campo de posibilidades para el mejoramiento del rendimiento de los alumnos y en lo que respecta a los aspectos cualitativos en la presentación de contenidos del currículo.

La RA encuentra otras aplicaciones en elárea de la ciencia, como por ejemplo, para la comprensión de la relación entre el mundo real (entornos y modelos tridimensionales) y las representaciones bidimensionales, que emplean con frecuencia modelos físicos para que los estudiantes puedan realizar sus bocetos desde distintos puntos de vista. La aparición de las tecnologías gráficas avanzadas está influyendo en la manera en que los usuarios pueden manipular la realidad, ofreciendo la posibilidad de sustituir modelos físicos o corpóreos por modelos virtuales, con los que se puede interactuar de forma intuitiva(De la Torre Cantero, Martin-Dorta, Pérez, Carrera, y González: 2015).

La utilización de modelos tridimensionales es ya habitualen otros ámbitos educativos, como por ejemplo, en biología o en las artes plásticas. En Pekín Cai, Wang y Chiang (2014) condujeron una investigación en la que se implementó la Realidad Aumentada en los procesos de enseñanza de la química para valorar su efecto en un grupo de estudiantes con bajo rendimiento. En este trabajo se propone desarrollar la investigación mediante la integración de un grupo de control para comparar los beneficios de la Realidad Aumentada respecto a otro grupo de estudio.

En el Instituto Tecnológico de Monterrey se puso en ejecución una aplicación de la RA y luego se evaluó la motivación que genera la herramienta en el aprendizaje de las matemáticas entre estudiantes de Ingeniería. Como se evidenció un alto valor agregado a nivel motivacional de los estudiantes (Salinas, González, Quintero, Ríos, Ramírez y Morales: 2013)

En el contexto anterior, este artículo muestra los resultados de la investigación sobre el efecto que tiene la tecnología de la RA en el aprendizaje de estudiantes de Educación Básica y precisar este efecto a través de una mejoría en el rendimiento académico. Concretamente se diseñó una experiencia didáctica con el tema de las Regiones Naturales de Colombia en la asignatura de Ciencias Sociales, considerando el desarrollo de los contenidos desde el punto de vista conceptual, procedimental y actitudinal. La hipótesis general que motivó el estudio es la de que la implementación de la herramienta didáctica de la Realidad Aumentad, mejora el aprendizaje $\mathrm{y}$ en consecuencia el rendimiento académico en estudiantes de Educación Básica.

\section{Metodología}

\subsection{Naturaleza del estudio}

El estudio realizado es de naturaleza descriptiva. El objetivo de la investigación pretende evaluar el efecto de la realidad aumentada (variable independiente) sobre el rendimiento de los estudiantes (variable dependiente). Para ello se establecieron dos grupos: uno de trabajo o experimental y uno de control. Dada esta configuración, el estudio puede plantearse como una investigación de tipo cuasi experimental.

\subsection{Población y muestra}

La investigación tuvo como escenario el colegio Virginia Gutiérrez de Pineda IED de Bogotá. La población estudiantil de los grados 4, 5, 6 y 7 estaba integrada para el momento de la recolección datos por 350 alumnos- La muestra se conformó de manera aleatoria y proporcional, sin ningún tipo de discriminación por componentes sociales, de 
raza, comportamiento o conflictos éticos, y estuvo integrada por 183 estudiantes, distribuidos en dos grupos: uno de control y otro de estudio.

\subsection{Instrumentos de recolección de información}

Los datos que constituyen la base del análisis se recolectaron por medio de instrumentos aplicados antes (prueba de entrada) y después (prueba de salida) de utilizar RA. Las variables a estudiar fueron la implementación de la RA (variable independiente) y aprendizaje (variable dependiente), para describir en qué medida se mejora el aprendizaje de los estudiantes en sus dimensiones conceptual, procedimental y actitudinal.

El instrumento de medida fue una encuesta elaborada por los autores y verificada por expertos en el tema. Su estructura se basa en la escala de Likert: respuestas cerradas, de tipo cuantitativo, con un valor entre 1 y 5 puntos; se aplicó en los grupos involucrados en el estudio para recoger datos respecto a las temáticas, experiencias y vivencias relacionados con las herramientas utilizadas para la enseñanza del tema de las regiones naturales colombianas.

Otra técnica utilizada fue la observación, que se sistematizó durante un acompañamiento constante en el desarrollo de la investigación con el objeto de vincular y ordenar las impresiones y actitudes de los grupos frente a las herramientas utilizadas para la enseñanza del tema de las regiones naturales.

\subsection{Procedimientos de análisis}

El procesamiento de datos se hizo a partir de la conformación de una base de datos en Excel con el objeto de proceder posteriormente al respectivo análisis; en el proceso se aplicaron fórmulas para calcular estadísticos descriptivos y posteriormente se generaron los gráficos de los resultados. Para la comprobación de la hipótesis se utilizó la prueba $\mathrm{t}$ para muestras relacionadas utilizando el software SPSS.

\section{Resultados}

Los resultados generales obtenidos luego de analizar y graficar los datos recolectados en la aplicación de la prueba de entrada y la prueba de salida sobre la implementación de la herramienta de RA y su influencia en el mejoramiento del aprendizaje, evidencian una diferencia positiva en los estudiantes del grupo de estudio, ya que los porcentajes de la prueba de salida son más altos que los de la prueba de entrada (ver figura 1). En el caso del grupo control, se observa que los resultados de la prueba de salida no tienen una diferencia significativa con respecto a los de la prueba de entrada (ver figura 2). En consecuencia, los resultados indican de modo general que la implementación de la herramienta RA favorece los procesos de aprendizaje.

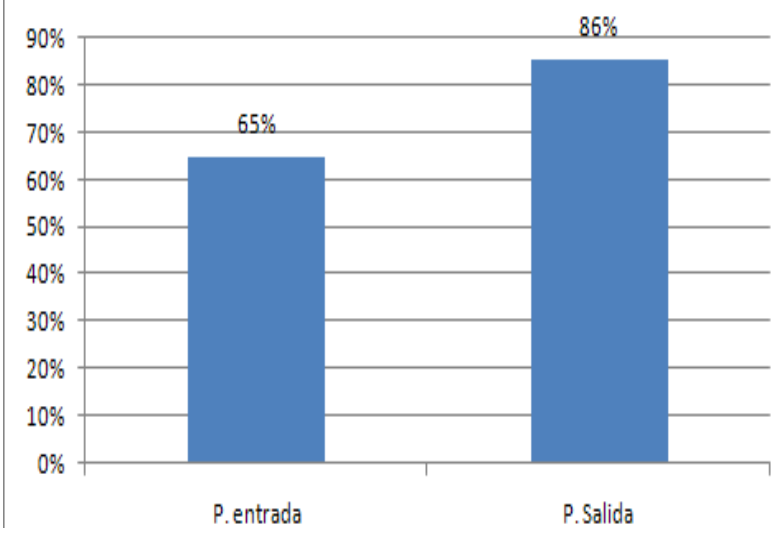

Figura 1

Puntuaciones de las pruebas de entrada y salida del grupo de estudio Fuente: proceso de investigación. 


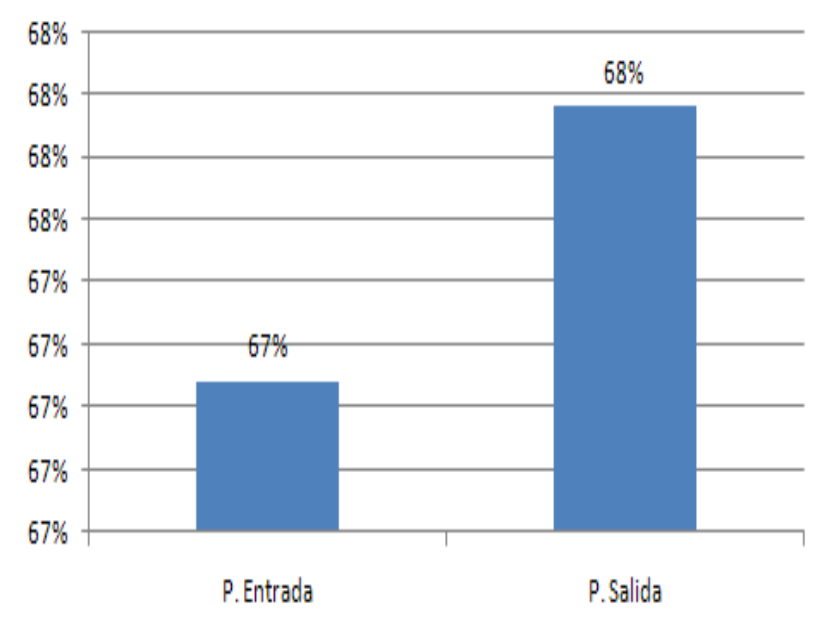

Figura 2

Puntuaciones de las pruebas de entrada y salida del grupo control Fuente: proceso de investigación.

Evaluación de diferencias estadísticamente significativas

Para la comprobación de la hipótesis, se hizo un análisis mediante la comparación de medias a partir de la Prueba $t$ de Student para muestras relacionadas; para ello se utilizó la herramienta de análisis de datos SPSS y se estableció el nivel de significancia para cada caso (grupo experimental y grupo de control), con el fin de generar la aceptación o rechazo de la hipótesis de acuerdo con la cual el aprendizaje de los estudiantes mejora cuando se aplica la herramienta de RA.

Dado que los datos obtenidos tienen una distribución normal, se aplicó la prueba t con dos muestras independientes para la comprobación de la hipótesis, utilizando una variable de agrupación denominada tratamiento. A esta variable se asignó una escala nominal que indica si un dato corresponde o no a la aplicación de la variable independiente. Se ingresaron al software 183 datos, correspondientes a la totalidad de la muestra. De ellos, 93 registros provienen del grupo de estudio, a quienes se les hizo el tratamiento o aplicación de la RA.

Los resultados obtenidos mediante el procesamiento de los datos a través del software SPSS, establecen que el nivel de significancia en la diferencia entre la prueba de entrada y salida en el grupo de estudio generó un valor de $\mathrm{p}=0,00<0.05$, lo cual evidencia que hay diferencias altamente significativas entre los dos momentos. Por lo tanto, se rechaza la hipótesis de igualdad de medias en favor de la hipótesis alternativa planteada por el investigador, es decir, se puede afirmar que la implementación de la herramienta de RA ha mejorado el aprendizaje entre los estudiantes con quienes se empleó la herramienta.

Cuando se observan los resultados de la significancia del grupo de control, no aparecen diferencias importantes entre los resultados previos y posteriores al trabajo con RA. El valor de significancia, en efecto, fue $p=0,391$, que es mayor que el nivel de significación 0,05 . Por lo tanto, este resultado confirma la aceptación de la hipótesis, es decir, en el grupo control no hubo diferencias en el rendimiento medio de los estudiantes.

\section{Conclusión}

A partir de la investigación desarrollada se confirmó la hipótesis de que el aprendizaje en los estudiantes mejora cuando se aplica la herramienta de realidad aumentada. Este resultado coincide con los de otras investigaciones y propuestas. En efecto, autores como De la Torre Cantero, MartínDorta, Pérez, Carrera y González (2015) también han encontrado que el rendimiento estudiantil se positivamente afectado luego de experiencias didácticas que incluyen el uso de la RA. En este estudio, las dos tecnologías utilizadas como alternativa a los modelos físicos fueron la realidad aumentada y tabletas digitales. Los resultados de la investigación muestran que ambas fueron valoradas positivamente por todos los usuarios, con valores medios de 7,10 y 8,00 (sobre 9).

Como conclusión general se puede establecer que la presente investigación ha evidenciado la importancia de herramientas que despierten el interés y el deseo de generar estructuras de conocimiento intencionadas y que al mismo tiempo favorezcan el trabajo colaborativo y la solución de problemas mediante procesos apropiados por parte de los estudiantes. 
Como principal aporte, se presenta la apertura hacia la apropiación e implementación de las herramientas de RA en los procesos educativos por parte de las estrategias aplicadas por los docentes en sus prácticas en el aula.

\section{Referencias}

Abud, M. (2012) MODELO DE OBJETOS DE APRENDIZAJE CON REALIDAD AUMENTADA. Revista Internacional de la Educación en Ingeniería, 5(1), 1-7

Basogain, X., Olabe, M., Espinosa, K., Rouèche, C., \&Olabe, J. C. (2007). Realidad Aumentada en la Educación: una tecnología emergente. Escuela Superior de Ingeniería de Bilbao, EHU. Recuperado de http://bit. ly/2hpZokY.

Bernat, A. \& Gros, B. (2008). Videojuegos y aprendizaje. Barcelona: Graó.

Cai, S. Wang, X. y Chiang, F (2014). A case study of Augmented Reality simulation system application in a chemistry course. Computers in Human Behavior 37, 31-40

Catalá, A.; Jaén, J. y Mocholí, J.A. (2008). Juegos ubicuos: experiencias de aprendizaje óptimas. En Gross Salvat, B. (ed.). Videojuegos y aprendizaje. Barcelona: Grao.

Cherrett, T., Wills, G., Price, J., Maynard, S.,\&Dror, I. (2009). Making training more cognitively effective: Making videosinteractive. British Journal of Educational Technology, 40 (6), 1124-1134

De Pedro, J. y Martínez, C.L. (2012). Realidad aumentada: una alternativa metodológica en la educación primaria nicaragüense.IEEE-RITA, 7(2), 102-108.

De la Torre Cantero, J., Martin-Dorta, N., Pérez, J. L. S., Carrera, C. C., \& González, M. C. (2015). Entorno de aprendizaje ubicuo con realidad aumentada y tabletas para estimular la comprensión del espacio tridimensional. Revista de Educación a Distancia, (37).

Fombona, J., Pascual, M.A. yMadeira, M.F. (2012). Realidad aumentada, una evolución de las aplicaciones de los dispositivos móviles. PíxelBit. Revista de medios y educación, 41, 197-210. Fundación Telefónica. (2012). Realidad Aumentada: una nueva lente para ver el mundo. Recuperado de: http://www.realidadaumentadafundaciontelefonica.com/realidad-aumentada. pdf

Rockwell, S. C., \& Singleton, L.A. (2007). The effect of the modality of presentation of streaming multimedia on information acquisition. Media Psychology, 9 (1), 179-191.

Salinas, P. González, E. Quintero, E. Ríos, H. Ramírez, H y Morales, S. (2013) The Development of a Didactic Prototype for the Learning of Mathematics Through Augmented Reality. ProcediaComputerScience 25 (2013), 62-70. doi: 10.1016/j.procs.2013.11.008

Suduc, A. M., Bizoi, M., Gorghiu, G., \&Gorghiu, L. M. (2010). Using web conferencing for disseminating the educational projects results. En H. Uzunboylu (Ed.), Innovation and Creativity in Education (pp 2813-2818). Amsterdam: ElsevierScience. 